\title{
A filament supported by different magnetic field configurations
}

\author{
Y. Guo ${ }^{1,2}$, B. Schmieder ${ }^{2}$, P. Démoulin ${ }^{2}$, T. Wiegelmann ${ }^{3}$, \\ G. Aulanier ${ }^{2}$, T. Török ${ }^{2}$, and V. Bommier ${ }^{4}$ \\ ${ }^{1}$ Department of Astronomy, Nanjing University, Nanjing 210093, China \\ email: guoyang@nju.edu.cn \\ ${ }^{2}$ LESIA, Observatoire de Paris, CNRS, UPMC, Université Paris Diderot, 5 place Jules \\ Janssen, 92190 Meudon, France \\ ${ }^{3}$ Max-Planck-Institut für Sonnensystemforschung, Max-Planck-Strasse 2, 37191 \\ Katlenburg-Lindau, Germany \\ ${ }^{4}$ LERMA, Observatoire de Paris, CNRS, UPMC, Université Paris Diderot, 5 place Jules \\ Janssen, 92190 Meudon, France
}

\begin{abstract}
A nonlinear force-free magnetic field extrapolation of vector magnetogram data obtained by THEMIS/MTR on 2005 May 27 suggests the simultaneous existence of different magnetic configurations within one active region filament: one part of the filament is supported by field line dips within a flux rope, while the other part is located in dips within an arcade structure. Although the axial field chirality (dextral) and the magnetic helicity (negative) are the same along the whole filament, the chiralities of the filament barbs at different sections are opposite, i.e., right-bearing in the flux rope part and left-bearing in the arcade part. This argues against past suggestions that different barb chiralities imply different signs of helicity of the underlying magnetic field. This new finding about the chirality of filaments will be useful to associate eruptive filaments and magnetic cloud using the helicity parameter in the Space Weather Science.
\end{abstract}

Keywords. Sun: corona, Sun: filaments, Sun: magnetic fields

\section{Introduction}

A filament or prominence is constructed by plasma that is typically a hundred times cooler and denser than the coronal surroundings. The dense plasma is usually thought to be supported by Lorentz force provided by dipped magnetic field lines. Magnetic dips could be present in quadrupolar fields (Kippenhahn \& Schlüter 1957), or in bipolar fields with a flux rope (Kuperus \& Raadu 1974; Aulanier \& Démoulin 1998), or highly sheared field lines below a strong arcade field (Antiochos et al. 1994). The flux rope model corresponds to the so-called inverse configuration ,i.e. the orientation of the magnetic field in the filament is inverse compared to the photospheric magnetic field, the later one is pointing from positive to negative polarity. The other two models correspond to the so-called normal configuration.

Magnetic field configurations play a key role in many aspects of a filament, such as its pattern, suspension, and formation. However, vector magnetic fields are currently not available for the chromosphere and the corona in practice. Therefore, these fields are usually extrapolated from the magnetic fields observed in the photosphere by the assumption of the force-free field model, i.e., $\nabla \times \mathbf{B}=\alpha \mathbf{B}$. The force-free parameter $\alpha$ usually changes in space in the corona with complicated magnetic field structures (Régnier et al. 2002; Schrijver et al. 2005), which yields a nonlinear force-free field (NLFFF). The gravity of the relatively denser plasma in a filament could affect the associated magnetic field 

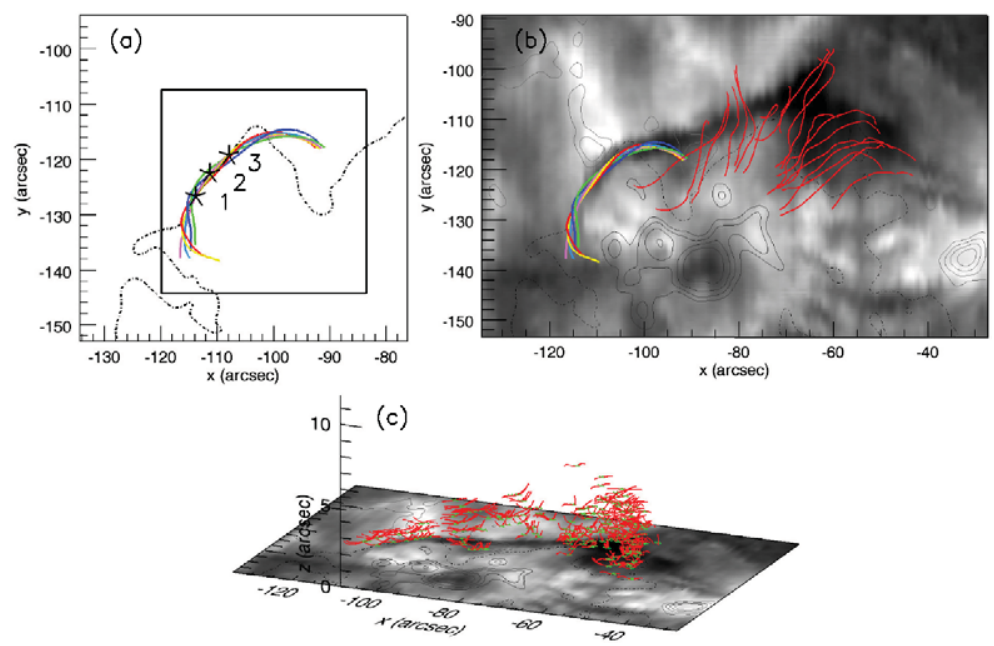

Figure 1. (a) Twisted field lines represent the flux rope. Dash-dotted line denotes the polarity inversion line. A third order polynomial is adopted to fit the polarity inversion line between points 1 and 3 to approximate the horizontal flux rope axis. The square is the projection of a rectangular box with $80 \times 80 \times 5$ grid points just above the bottom layer. (b) $\mathrm{H} \alpha$ filament overlaid with the flux rope and some selected field lines. (c) Dips ("+" signs) and field line sections in the extrapolated magnetic field.

configuration. However, the force-free field model may be still adequate in active regions, since the magnetic field strength in active region filaments could be very large, which is up to 600-700 G for instance (Kuckein et al. 2009).

Guo et al. (2010) performed a NLFFF extrapolation for the active region NOAA 10767 using Wiegelmann's method (2004). An $\mathrm{H} \alpha$ filament was observed in the active region. A magnetic flux rope was found by the NLFFF extrapolation corresponding to the eastern part of the filament, while magnetic dips of arcade field lines coincided with the other part. In this paper, we study how different grid resolutions in the optimization algorithm affect the extrapolation results in the definition of the flux rope. Four cases, where the spatial resolution along the height are 2, 1, $\frac{1}{2}$ and $\frac{1}{4}$ times that along the horizontal plane, have been carefully tested with Low and Lou's analytical NLFFF solution and applied to the active region NOAA 10767.

\section{Analysis and Results}

Active region NOAA 10767 was observed by Télescope Héliographique pour l'Etude du Magnétisme et des Instabilités Solaires/Multi-Raies (THEMIS/MTR) from 9:54 to 10:41 UT on 2005 May 27. The Stokes profiles obtained by the Fe $6302.5 \AA$ line are fitted by the inversion code UNNOFIT to infer the vector magnetic field (Landolfi \& Landi Degl'Innocenti 1982; Bommier et al. 2007). Due to the fact that opposite orientations of the transverse magnetic field generate indistinguishable linear polarizations, a $180^{\circ}$ ambiguity in the direction of the transverse field is present. We remove the $180^{\circ}$ ambiguity in two steps. First, it is removed preliminarily by an automatic algorithm, the nonpotential magnetic field calculation method (Georgoulis 2005). Then, some regions with larger current borders, where transverse fields change abruptly, are reconsidered by an interactive code by removing the ambiguity manually. In order to simulate a force-free 
Table 1. Metrics for NLFFF Extrapolations from the Observation Data

\begin{tabular}{|c|c|c|c|}
\hline Grid Resolution ${ }^{1}$ & $E / E_{\text {pot }}^{2}$ & $<\mathrm{CW} \sin \theta>^{3}$ & $<\left|f_{i}\right|>^{4}$ \\
\hline$\Delta z=2 \Delta x=2 \Delta y$ & 1.41 & 0.28 & $4.5 \times 10^{-3}$ \\
\hline$\Delta z=\Delta x=\Delta y$ & 1.36 & 0.28 & $4.9 \times 10^{-3}$ \\
\hline$\Delta z=\frac{1}{2} \Delta x=\frac{1}{2} \Delta y$ & 1.30 & 0.28 & $5.2 \times 10^{-3}$ \\
\hline$\Delta z=\frac{1}{4} \Delta x=\frac{1}{4} \Delta y$ & 1.25 & 0.26 & $5.6 \times 10^{-3}$ \\
\hline
\end{tabular}

Notes:

1 The optimization codes with different grid resolutions.

2 Energy contained in the NLFFF referred to that in the potential field.

${ }^{3}<\mathrm{CW} \sin \theta>=\frac{\sum_{i} J_{i} \sin \theta_{i}}{\sum_{i} J_{i}}$, where $\sin \theta_{i}=\frac{\left|\mathbf{J}_{i} \times \mathbf{B}_{i}\right|}{J_{i} B_{i}}$.

${ }^{4}\left|f_{i}\right|=\left|(\nabla \cdot \mathbf{B})_{i}\right| /\left(6 B_{i} / \Delta x\right)$. All the metrics are calculated in the selected volume in Figure 1 a.

and torque-free boundary on the bottom layer, we preprocess the boundary data by minimizing the total magnetic force and torque and smoothing small scale structures.

The preprocessed bottom boundary are used by the optimization code to extrapolate the NLFFF (Wheatland et al. 2000; Wiegelmann 2004). Selected field lines are plotted in Figure 1. The magnetic field configurations associated with the $\mathrm{H} \alpha$ filament are partly flux rope and partly arcade as shown in Figure 1b. The magnetic dips of these two field configurations are thought to support the filament material (Figure 1c). The local magnetic helicity sign in this region is negative, as suggested by the left-handed flux rope and the left-skewed arcades. However, the filament barb chiralities are different, which is right-bearing in the flux rope part and left-bearing in the arcade part (refer to Guo et al. (2010) for analysis in detail).

We further test the extrapolation results with codes of different spatial resolutions in $z$, which is along the height. The horizontal resolutions keep unchanged and equal to each other. To test the performance of these codes $(\Delta z=2 \Delta x=2 \Delta y, \Delta z=\Delta x=\Delta y$, $\Delta z=\frac{1}{2} \Delta x=\frac{1}{2} \Delta y$, and $\Delta z=\frac{1}{4} \Delta x=\frac{1}{4} \Delta y$ ), we prepare four analytical solutions (Low $\&$ Lou 1990) with the four different spatial resolutions. The initial condition is specified by the potential field extrapolated from the bottom boundary. If all the six boundaries are specified, the analytical NLFFF can be recovered to a very good degree. If only the bottom boundary is specified, and the lateral and top boundaries are given by the potential field extrapolated from the bottom boundary, all the codes can still recover the NLFFF in the center of the computing volume to an acceptable degree.

We apply the four versions of the optimization procedure to the preprocessed vector magnetic field observed by THEMIS/MTR. Table 1 shows that the metrics of the nonlinear force-free fields extrapolated by all the codes do not differ from each other too much. We decompose the magnetic field components in a local reference frame of the flux rope and study their distributions in Figure 2. We find that $B_{v}$ always changes its sign when it crosses a defined axis, which indicates the existence of twisted field lines of a flux rope. Two cases with grid resolution of $\Delta z=2 \Delta x=2 \Delta y$ and $\Delta z=\Delta x=\Delta y$ give similar heights of the flux rope axis. However, the other two codes $\left(\Delta z=\frac{1}{2} \Delta x=\frac{1}{2} \Delta y\right.$, and $\Delta z=\frac{1}{4} \Delta x=\frac{1}{4} \Delta y$ ) cannot recover the flux rope to the same physical height, but only the same grid points as shown in Figure 2.

\section{Conclusions}

The chirality of a filament barb depends on both the magnetic helicity (positive/negative) and the magnetic field configuration (flux rope/arcade). Based on the NLFFF extrapo- 

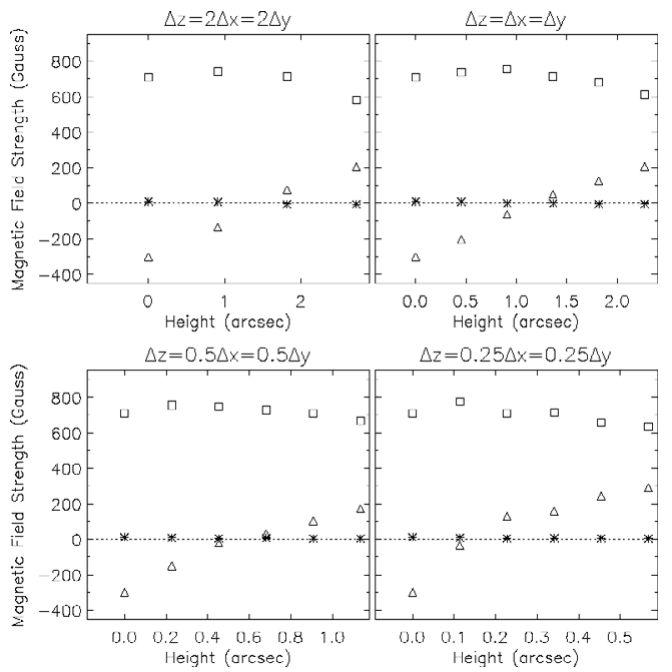

Figure 2. Distributions of $B_{p}$ (square), $B_{v}$ (triangle), and $B_{z}$ (asterisk) along the height at the point 2 (Figure 1a). $B_{p}$ and $B_{v}$ are the horizontal components, parallel and orthogonal to the axis of the flux rope, respectively. Different panels show the results obtained with the codes of different grid resolutions.

lation of the active region NOAA 10767 magnetic field observed by THEMIS/MTR on 2005 May 27, we find that the chirality of filament barbs in different sections are different, i.e., right-bearing in the flux rope part and left-bearing in the arcade part, although the magnetic helicity (negative, which is equivalent to dextral axial field chirality) is the same along the whole filament.

The existence of the flux rope is further confirmed by extrapolations with four codes of different grid resolutions along the height. However, we cannot get more grid points to resolve the flux rope along height when the resolution is increased. We think this problem is caused by the boundary conditions. First, we do not have data on the full boundaries, such as the lateral and top surfaces for a box, which are specified by a potential field extrapolation instead. Secondly, As a prior requirement for the preprocessing, the bottom boundary should be well isolated and flux balanced, which is not fulfilled due to the limitation of the field of view in the observation. Appropriate preprocessing of boundary conditions is crucial for a credible extrapolation.

\section{References}

Antiochos, S. K., Dahlburg, R. B., \& Klimchuk, J. A. 1994, Astrophys. J. Lett., 420, L41

Aulanier, G. \& Démoulin, P. 1998, Astron. Astrophys, 329, 1125

Bommier, V., Landi Degl'Innocenti, E., Landolfi, M., \& Molodij, G. 2007, Astron. Astrophys, 464,323

Georgoulis, M. K. 2005, Astrophys. J. Lett., 629, L69

Guo, Y., Schmieder, B., Démoulin, P., Wiegelmann, T., Aulanier, G., Török, T., \& Bommier, V. 2010, Astrophys. J., 714, 343

Kippenhahn, R. \& Schlüter, A. 1957, Zeitschrift fur Astrophysik, 43, 36

Kuckein, C., Centeno, R., Martínez Pillet, V., Casini, R., Manso Sainz, R., \& Shimizu, T. 2009, Astron. Astrophys, 501, 1113

Kuperus, M. \& Raadu, M. A. 1974, Astron. Astrophys, 31, 189 
Landolfi, M. \& Landi Degl'Innocenti, E. 1982, Solar Phys., 78, 355

Low, B. C. \& Lou, Y. Q. 1990, Astrophys. J., 352, 343

Régnier, S., Amari, T., \& Kersalé, E. 2002, Astron. Astrophys, 392, 1119

Schrijver, C. J., De Rosa, M. L., Title, A. M., \& Metcalf, T. R. 2005, Astrophys. J., 628, 501

Wheatland, M. S., Sturrock, P. A., \& Roumeliotis, G. 2000, Astrophys. J., 540, 1150

Wiegelmann, T. 2004, Solar Phys., 219, 87 